\title{
Efficacy of multimodal anticancer therapy in the course of pancreatic neuroendocrine carcinoma
}

\author{
Helena Zwinczewska ${ }^{11,2}$, Marta Opalinska², Anna Sowa-Staszczak@1, 2, Anna Kurzynska ${ }^{1,2}$, \\ Agnieszka Stefanska², Alicja Hubalewska-Dydejczyk ${ }^{(1,2}$
}

${ }^{1}$ Department of Endocrinology, University Hospital, Krakow, Poland

${ }^{2}$ Jagiellonian University Medical College, Krakow, Poland

Key words: neuroendocrine tumours; PRRT; $P N E C$; somatostatin analogue

Neuroendocrine neoplasms (NENs) are a heterogeneous group, comprising well-differentiated neuroendocrine tumours (NETs) and poorly differentiated neuroendocrine carcinomas (NECs). NETs and NECs show major differences regarding histology, genetics, biological behaviour, and clinical manifestations [1]. The malignancy of NECs is high, which explains the poor prognosis. NECs are relatively rare; therefore, few data on long-term management are available. The present study shows the 10-year follow-up of a patient diagnosed with a disseminated pancreatic neuroendocrine carcinoma ( $\mathrm{pNEC}$ ) in young age.

In 2008, a 28-year-old Caucasian male with a one-year history of abdominal pains and with no concomitant diseases was admitted to the hospital due to acute pancreatitis. On CT examination, an 11-mm cyst in the tail of the pancreas was described. Due to persistent pain in the epigastrium, accompanied by nausea and vomiting, magnetic resonance imaging was performed revealing a pancreatic tail tumour $54 \times 42 \mathrm{~mm}$, suspected to be adenocarcinoma infiltrating splenic vessels and adipose tissue. In addition, metastatic lymph nodes and 2 liver metastases in segments IV and VIIII were described. A liver biopsy confirmed NEN with chromogranin expression. The pancreatic biopsy was non-diagnostic. Somatostatin receptor imaging (SRI) showed tracer uptake in the primary lesion and hepatic metastases. Subtotal left-sided pancreatectomy was performed in April 2009 (histopathologically pNEC, pT4N2M1, Ki-67 50\%, lymph node metastases 17/20).

One month later, thermoablation of only one available liver metastasis was performed. Due to very good SSTR expression in the metastatic lesions and the oncologist's suggestion, the patient was qualified for Peptide Receptor Radionuclide Therapy (PRRT).
In June 2009 the patient received $740 \mathrm{MGq}$ of $\left[{ }^{90} \mathrm{Y}\right]$ Y-DOTA-TATE without complication with concomitant chemotherapy (gemcitabine-erlotinib) from June 2009 to August 2011, giving stabilisation of the disease in consecutive follow-up CT scans.

In February 2013, SRI revealed an increased tracer uptake in the retropharyngeal lymph node on the right side. The repeated biopsies of retropharyngeal lymph node were negative.

In June $2016\left[{ }^{18} \mathrm{~F}\right] \mathrm{F}-\mathrm{FDG}-\mathrm{PET} / \mathrm{CT}$ detected metabolically active infiltration to cervical lymph nodes invading adherent soft tissues.

In September 2016, due to further progression of disease, a paraspinal lump at the base of the skull was removed (histopathologically a metastasis of pancreatic NET, Ki67 3\%).

Post-operative SRI in November 2016 showed new metastatic lesions in both liver lobes, which disqualified the patient from radical treatment and surgical interventions. The retropharyngeal lymph node was still present. Treatment with a long-acting somatostatin analogue was initiated in December 2016. Since Jan 2017, the patient has additionally received second-line chemotherapy (capecitabine + temozolomide). So far, 42 cycles of systemic treatment have been administered, with good tolerance of treatment. Since then, no disease progression has been reported.

pNECs are rare tumours, with an incidence of 10-20\% according to previous WHO classification (2010), which did not discriminate between pNECs and NET G3. Curative surgery is the first line of treatment, but it is difficult to achieve satisfactory results with this approach alone [1]. Moreover, pNEC patients often present initially advanced, disseminated stages of disease and are not eligible for surgical treatment [2]. PRRT has proved 


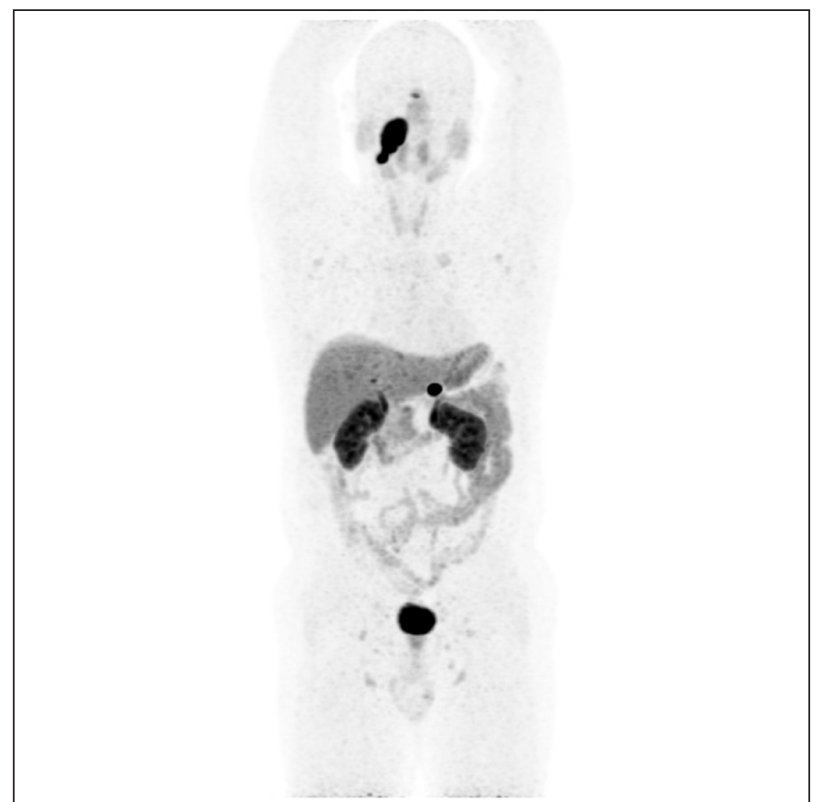

Figure 1. Very good expression of SSTR in cervical lymph nodes with infiltration of the levator veli palatini, sphincter muscle, the posterior wall of the nasopharynx, and pharyngeal tonsils seen in $\left[{ }^{68} \mathrm{Ga}\right] \mathrm{Ga}$-DOTA-TATE PET/CT in 2016 before onset of CAPTEM chemotherapy

to be an effective and well-tolerated treatment modality in patients with pNETs, possibly also in stage G3 [3]. Other treatment patterns are based on multi-agent chemotherapy used in small cell lung carcinoma [1,4]. Recommended in pNETs, G3 regimens have changed over the years. Current therapeutic options include streptozotocin and 5-fluorouracil or capecitabine with temozolomide. In NECs, platinum-based combinations are widely used as first-line treatment. Rogowski et al. reported that the combination of capecitabine and temozolomide is an effective treatment for patients with NET G3 with high Ki-67 index [5]. This regimen was used in the presented case as a second-line treatment. Survival of patients with advanced stages of disease is estimated in months. In the presented case radical treatment was not possible due to the stage of disease. Despite being NEC (according to WHO 2010 classification), the primary tumour had a very good SSTR expression. Montanier reported a case of pNEC with liver metastases with low mitotic index and a very high Ki-67 index $(45-70 \%)$ treated with PRRT([177 Lu] Lu-DOTATATE), which allowed for 3 years of complete remission [6]. In the presented case the progression-free period equalled 4 years, but the patient received chemotherapy for half of this time. The retropharyngeal lymph node metastasis was identified as a distant metastasis of pancreatic NET, but it was significantly different from the primary lesion. The long course of the disease and good SSTR expression may raise doubts about whether

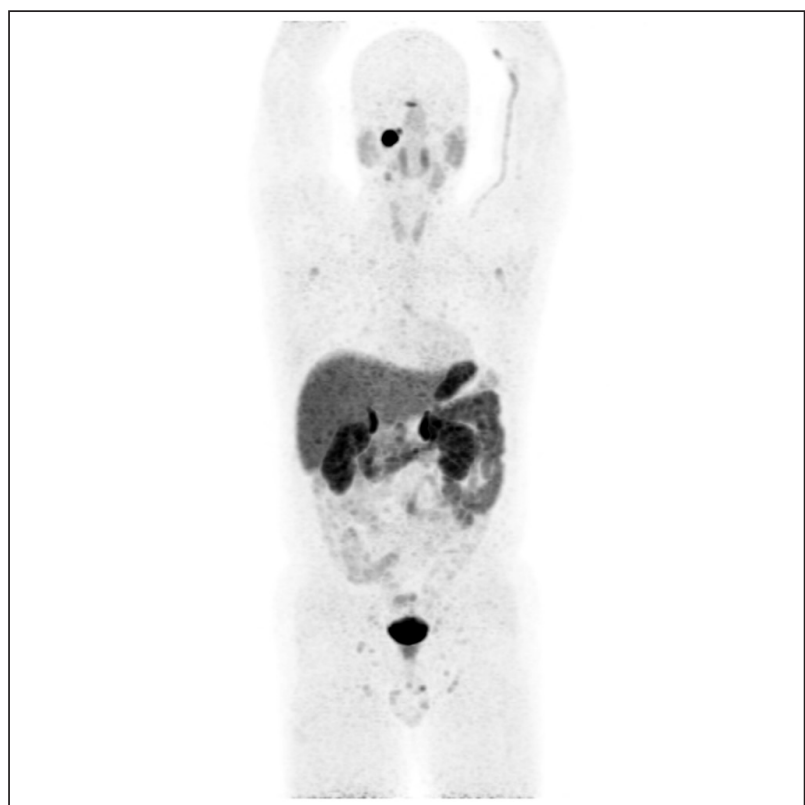

Figure 2. Very good expression of SSTR in cervical lymph nodes with infiltration of the levator veli palatini, sphincter muscle, the posterior wall of the nasopharynx, and pharyngeal tonsils seen in $\left[{ }^{68} \mathrm{Ga}\right] \mathrm{Ga}$-DOTA-TATE PET/CT in 2020 after 12 years of multimodality treatment

in our case NEC could be NET G3 according to the current WHO classification (2019). To our knowledge, no such case was previously described in the literature.

The use of PRRT with subsequent chemotherapy and treatment with long-acting somatostatin analogue proved to be effective in the presented case, confirming the benefit from multimodal anticancer therapy. There is a need to use all diagnostic methods to select the optimal therapy in patients with pNECs, to provide them with better care and the longest possible survival.

\section{References}

1. Zhang MY, He Du, Zhang S. Pancreatic neuroendocrine tumors G3 and pancreatic neuroendocrine carcinomas: Differences in basic biology and treatment. World J Gastrointest Oncol. 2020; 12(7): 705-718, doi: 10.4251/wjgo.v12.i7.705, indexed in Pubmed: 32864039.

2. Lokesh KN, Anand A, Lakshmaiah KC, et al. Clinical profile and treatment outcomes of metastatic neuroendocrine carcinoma: A single institution experience. South Asian J Cancer. 2018; 7(3): 207-209, doi: 10.4103/sajc.sajc_176_17, indexed in Pubmed: 30112343.

3. Lorenzoni A, Capozza A, Artale S, et al. Impressive Response to Tandem Treatment With [90Y]DOTATOC and [177Lu]DOTATOC in Grade 3 Pancreatic Neuroendocrine Carcinoma. Clin Nucl Med. 2018; 43(7): 506-508, doi: 10.1097/RLU.0000000000002116, indexed in Pubmed: 29742602.

4. Alese OB, Jiang R, Shaib W, et al. High-Grade Gastrointestinal Neuroendocrine Carcinoma Management and Outcomes: A National Cancer Database Study. Oncologist. 2020; 25(5): e877, doi: 10.1634/theoncologist.2020-0135, indexed in Pubmed: 32395915.

5. Rogowski W, Wachuła E, Gorzelak A, et al. Capecitabine and temozolomide combination for treatment of high-grade, well-differentiated neuroendocrine tumour and poorly-differentiated neuroendocrine carcinoma - retrospective analysis. Endokrynol Pol. 2019; 70(4): 313-317, doi: 10.5603/EP.a2019.0010, indexed in Pubmed: 30843182

6. Montanier N, Joubert-Zakeyh J, Pétorin C, et al. The prognostic influence of the proliferative discordance in metastatic pancreatic neuroendocrine carcinoma revealed by peptide receptor radionuclide therapy: Case report and review of literature. Medicine (Baltimore). 2017; 96(6): e6062, doi: 10.1097/MD.0000000000006062, indexed in Pubmed: 28178157. 